\section{Credit: JESPER KLAUSEN/SCIENCE PHOTO LIBRARY/Getty}

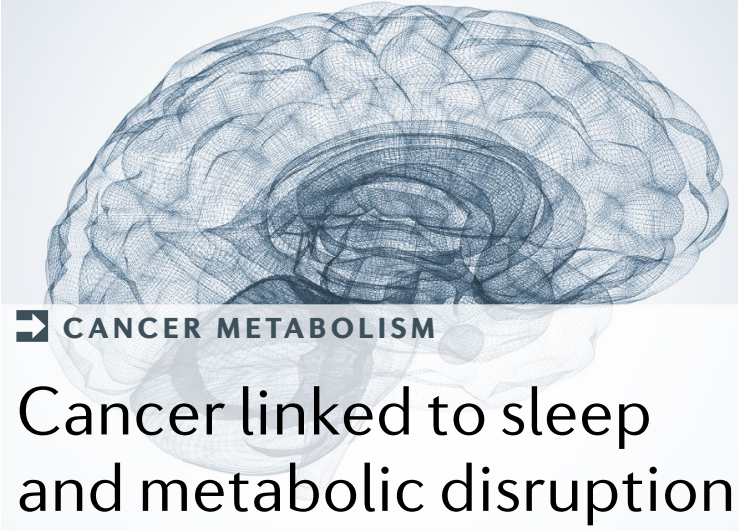

Patients with cancer often suffer from sleep and metabolic problems, but the mechanistic basis for these issues is unclear. Now, new research has directly linked cancer to both sleep disruption and metabolic dysregulation.

In a mouse model of nonmetastatic breast cancer, Jeremy Borniger, William Walker II and colleagues show that cancer alters satiety hormone signalling. This change disrupts central hypocretinproducing neurons, which results in

can alter sleep and metabolism sleep and metabolic dysregulation via the sympathetic nervous system.

"Prior research has shown that cancer can promote an inflammatory state that may promote sleep and metabolic problems; however, although we found evidence of inflammation in tandem with sleep and metabolic disruption in our model of nonmetastatic breast cancer, blocking inflammation did not rescue these problems," explains Borniger. "We therefore investigated neural populations that couple sleep and metabolism, and as hypocretinproducing neurons have been widely studied, we started there."

The authors began by measuring changes to sleep during the course of tumour development in their mouse model using electroencephalogram and electromyogram telemetry. Borniger explains that in their experiments they used non-metastatic breast cancer cells (67NR) to prevent brain or elsewhere and promoting sleep and/or metabolic problems. They then examined inflammation cancer cells metastasizing to the

in the periphery and brain; glucose, lactate and pyruvate tolerance; and the activity of hypocretin-producing neurons and melanin-concentrating hormone-producing neurons.

"The primary finding was that non-metastatic tumours alone can alter sleep and metabolism by first altering leptin and ghrelin signalling, which sets off a cascade of events that promote sleep and metabolic dysregulation," adds Borniger.

"Our data provide a holistic view of tumour-brain-liver communication that mediates multiple aspects of cancer co-morbidities.'

The authors hope that with further research they will be able to learn more about how hypocretin-producing neurons are linked to abnormalities in sleep and metabolism in cancer and define whether this pathway is conserved in humans.

Alan Morris

\title{
Redefining metabolically healthy obesity
}

New research in a large cohort of women demonstrates that obesity is a risk factor for cardiovascular disease (CVD), irrespective of whether the individual remained metabolically healthy over long periods of time.

"There is an ongoing debate regarding whether there is a subgroup of patients with obesity without an increased risk of major chronic diseases such as CVD," explains author Matthias Schulze. Previous work had indicated that being metabolically healthy might be a transient phenotype in many patients with obesity; however, this theory has not been confirmed by definitive long-term studies, leading Schulze and colleagues to carry out the current study.

The study included 90,257 women from the Nurses' Health Study who were recruited in 1976 and followed up every 2 years from 1980 to 2010 . The women were stratified by BMI category and metabolic health, with 'metabolically healthy' being defined as an absence of diabetes mellitus, hypertension and hypercholesterolaemia. At each follow-up, BMI was recalculated and incident cases of CVD (myocardial infarction and/or stroke) were recorded.

As would be expected, the women with obesity who were metabolically unhealthy at baseline or who converted to a metabolically unhealthy phenotype during follow-up had the highest risk of CVD. Interestingly, the researchers found that women with obesity who maintained a metabolically healthy phenotype during the follow-up still had an increased risk of CVD compared with women with a stable normal weight who were metabolically healthy. In addition, most of the women (across all BMI categories) who were metabolically healthy at baseline transitioned to a metabolically unhealthy phenotype during follow-up.

The authors note that their study is limited by only including women, who were mainly of white European origin.

\section{ce \\ there might be alternative ways to define metabolic} health

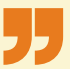

ORIGINAL ARTICLE Borniger, J. C. et al. A role for hypocretin/orexin in metabolic and sleep abnormalities in a mouse model of non-metastatic breast cancer. Cell Metab. https://doi.org/ 10.1016/j.cmet.2018.04.021(2018)

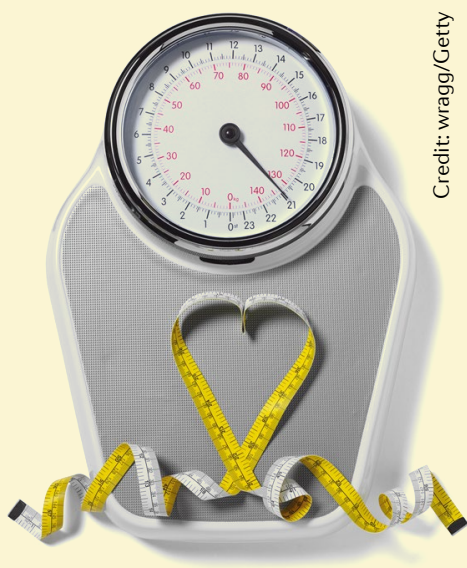

However, "there might be alternative ways to define metabolic health that potentially could identify obese subgroups that are not at increased risk of CVD," concludes Schulze. "Such alternatives should be explored in more detail."

Claire Greenhill

ORIGINAL ARTICLE Eckel, N. et al. Transition from metabolic healthy to unhealthy phenotypes and association with cardiovascular disease risk across BMI categories in 90,257 women (the Nurses' Health Study): 30 year follow-up from a prospective cohort study. Lancet Diabetes Endocrinol. https://doi.org/10.1016/S22138587(18)30137-2 (2018) 